\title{
GLAD!
}

Revue sur le langage, le genre, les sexualités

$01 \mid 2016$

Varia

\section{GLAD! revue féministe et indisciplinée}

Un projet scientifique, éditorial et politique

GLAD! a Feminist and Undisciplined Journal. An Academic Project, an Editorial

Endeavour, a Political Perspective

Julie Abbou, Maria Candea, Alice Coutant, Mona Gérardin-Laverge, Stavroula Katsiki, Noémie Marignier, Lucy Michel et Charlotte Thevenet

\section{OpenEdition}

\section{Journals}

Édition électronique

URL : http://journals.openedition.org/glad/260

DOI : $10.4000 /$ glad.260

ISSN : 2551-0819

Éditeur

Association GSL

\section{Référence électronique}

Julie Abbou, Maria Candea, Alice Coutant, Mona Gérardin-Laverge, Stavroula Katsiki, Noémie Marignier, Lucy Michel et Charlotte Thevenet, «GLAD! revue féministe et indisciplinée », GLAD! [En ligne], 01 | 2016, mis en ligne le 15 novembre 2016, consulté le 17 décembre 2020. URL : http:// journals.openedition.org/glad/260 ; DOI : https://doi.org/10.4000/glad.260

Ce document a été généré automatiquement le 17 décembre 2020.

\section{(c) $(1)$}

La revue GLAD! est mise à disposition selon les termes de la Licence Creative Commons Attribution -

Pas d'Utilisation Commerciale - Pas de Modification 4.0 International. 


\title{
GLAD! revue féministe et indisciplinée
}

\author{
Un projet scientifique, éditorial et politique \\ GLAD! a Feminist and Undisciplined Journal. An Academic Project, an Editorial \\ Endeavour, a Political Perspective
}

Julie Abbou, Maria Candea, Alice Coutant, Mona Gérardin-Laverge, Stavroula Katsiki, Noémie Marignier, Lucy Michel et Charlotte Thevenet

$1 \quad$ GLAD! Revue sur le langage, le genre, les sexualités est une nouvelle revue consacrée aux travaux scientifiques, artistiques et politiques articulant recherches sur le genre et les sexualités et recherches sur le langage d'un point de vue critique. Elle se veut un espace de discussion et de rencontre entre des travaux, des angles d'attaque et des pratiques multiples. C'est aussi un espace de traduction, d'exploration de la langue et de réflexion critique sur les critères de légitimité linguistique et académique.

2 À l'occasion de ce numéro inaugural, nous revenons dans cette introduction sur la volonté à la fois intellectuelle et politique qui a présidé à la création d'un tel espace, sur le contexte académique dans lequel la revue a émergé, sur les questions que soulève aujourd'hui la rencontre entre genre, sexualités et langage depuis différents prismes disciplinaires, ainsi que sur les enjeux éditoriaux qui ont façonné la revue. Nous présenterons enfin les travaux que nous avons choisi de publier dans ce premier numéro.

\section{Un projet intellectuel et politique : pour une approche critique du genre et du langage}

3 L'unité et la spécificité de la revue tiennent en premier lieu à sa thématique, qui articule une perspective langagière à un ancrage théorique en études de genre et des sexualités, chacun de ces éléments pouvant servir de cadre épistémologique. L'articulation de ces deux perspectives repose sur une approche fondamentalement critique et politique. 
4 Par compréhension critique du langage, nous entendons une appréhension de celui-ci comme un matériau complexe, c'est-à-dire non transparent, polysémique et hétérogène. Les études de genre, les études post/décoloniales et les études féministes permettent en effet de revisiter les discours, les structures linguistiques et leurs interactions à la lumière de l'idéologie et des rapports sociaux. Elles montrent comment ces matériaux langagiers sont façonnés par les rapports de pouvoir, de domination et de subversion, mais aussi comment ces mêmes matériaux agissent en retour sur les idéologies. Elles ouvrent également la voie à une réflexion sur le caractère situé de la production du savoir, qui permet de repenser les théories de la langue et du discours comme nécessairement ancrées dans leur contexte de production, mais aussi de mettre au centre les productions marginales ou marginalisées. Poser un tel regard critique et politique sur la constitution des disciplines permet de renouveler notre outillage analytique. Cette conception nourrit et prolonge les approches dialogiques du langage compris comme un espace constitutif du social.

5 Une compréhension critique du genre et des sexualités implique, quant à elle, de saisir les différents discours sur le genre et les sexualités dans leurs dimensions et leurs effets à la fois matériels et idéologiques. La revue adopte un positionnement résolument constructiviste dans son approche du genre et cherche à remettre en question les logiques essentialisantes à l'œuvre dans l'analyse théorique et dans la société. En saisissant le genre lui-même comme un processus de catégorisation, et en abordant les relations que ce processus entretient avec les différentes idéologies du genre, il s'agit de mettre au jour les matrices discursives et linguistiques de la construction du genre et de retracer les généalogies de la bicatégorisation à la lumière des rapports de pouvoir et de résistance. À ce titre, les pratiques discursives des groupes et/ou sujets minorisés (du point de vue du sexe, du genre, de la sexualité) - en tant qu'elles créent des troubles dans le genre et reconfigurent les rapports de pouvoir - sont aussi centrales pour la revue que l'analyse critique des processus de domination. Enfin, une approche critique du genre et des sexualités est inséparable d'une compréhension critique des autres rapports sociaux, notamment de classe, de race, d'âge et de colonisation, nécessaire pour lire le social.

\section{État des lieux des recherches sur le genre et le langage, une généalogie transdisciplinaire}

6 Le projet de la revue s'inscrit dans une généalogie transdisciplinaire. Aux prises avec la dimension idéologique du genre, les études féministes et les études sur le genre se sont en effet depuis longtemps confrontées à la question du rôle du langage dans la construction du genre et des sexualités : comment le genre signifie-t-il ? Quels sont la portée et les mécanismes de cette signification? Comment sont construites et se construisent les sexualités dans/par/hors les discours?

7 Pourtant, bien qu'un certain nombre de textes reconnaissent régulièrement l'importance de la langue, ou du moins d'une dimension sémiotique dans la construction du genre et des sexualités (Scott 1986, Butler 1990, Wittig 2001, Delphy 2008, Viennot 2014), ces lectures n'assortissent que rarement leur réflexion d'un travail sur la matérialité textuelle et langagière dans ses spécificités. En retour, les disciplines du langage ignorent souvent les enjeux liés au genre, et les recherches portant sur ces 
questions restent la plupart du temps à la marge, comme si elles outrepassaient leur cadre disciplinaire en portant leur regard sur des objets non traditionnels. Cet état des lieux semble dessiner un rendez-vous manqué entre les recherches sur le langage et les recherches sur le genre et les sexualités, qu'il est cependant nécessaire de contextualiser à deux égards.

Tout d'abord, un coup d'œil sur la production internationale concernant ces questions nous informe rapidement que la rencontre a bien eu lieu, principalement dans le monde anglophone, mais aussi dans les espaces lusophones, germanophones, etc. Le champ des Gender and Language Studies s'y est fortement développé depuis plusieurs décennies et compte de nombreux travaux de référence (en témoigne le nombre important de readers, par exemple Cameron 1990, 1992, Livia \& Hall 1997, Coates \& Pichler 2011, Eckert \& McConnell-Ginet 2003, Holmes \& Meyerhoff 2003, Mills 2008, Baker 2008, Cameron \& Kulick 2006, Talbot 2010, Motschenbacher 2010), de nombreux cursus et parcours de formation (à l'Université de Lancaster et à l'Université de Californie à Santa Barbara, pour ne citer que ceux-là), des événements scientifiques d'ampleur (notamment la conférence biennale de l'IGALA : l'International Gender and Language Association) et enfin une activité éditoriale riche autour de différentes revues internationales comme Gender \& Language, Signs, Women and Language, etc. Autant d'éléments qui témoignent de la vivacité et de la pleine reconnaissance de ce domaine de recherche. Le paysage francophone apparait ainsi en décalage par rapport à cette production mondiale qui saisit ensemble, depuis plus de quarante ans, les questions de genre et de langage.

9 Cependant, il faut encore relativiser cette exception francophone. En sciences du langage, dès les années 1980, des travaux sont publiés en France sur ces questions (Guiraud 1978, Ribery 1981, Violi 1987, Houdebine 1989, Yaguello 1989), mais ils restent clairsemés et n'émanent que d'une poignée de chercheur.es durant les deux décennies qui vont suivre. Dans les années 2000, le paysage s'étoffe un peu: des ouvrages paraissent (Armstrong, Bauvois \& Beeching 2001, Khaznadar 2002, Mathieu 2002, Michard 2002, Baider 2004, Bailly 2008, Michard \& Ribery 2008), des thèses sont soutenues (Breysse 2002, Abbou 2011, Perry 2011), des états de l'art sont réalisés (Michard \& Viollet 1991, Houdebine 2003, Arnold 2008, ou plus récemment Greco 2014), des actes de conférences publiés (Perry 2004) et c'est également la grande période des guides de féminisation, dont la liste serait trop longue à fournir. Mais il est encore difficile de voir là un champ d'étude.

C'est à partir des années 2010 que l'on peut commencer à parler d'un domaine de recherche à part entière. Différentes initiatives, des colloques et des journées d'études sont organisés tous les ans (Paris III 2009, Chypre 2010, Paris III 2012, Toulouse 2012, Saint-Étienne 2013, Montpellier 2014, Lyon 2015, Paris III 2015, Metz 2015, Paris III 2016). Ces événements vont permettre des rencontres et la mise en place de nouvelles dynamiques. Surtout, ils révèlent la diversité des approches théoriques : si des travaux s'intéressent toujours aux dispositifs de désignation des femmes et des hommes, et aux parlers des femmes et des hommes, on voit également émerger de nombreuses recherches qui s'intéressent à la construction du genre lui-même à travers les discours, et aux phénomènes socio-discursifs et linguistiques de catégorisation des masculinités et des féminités. Le champ passe ainsi de la question des femmes à la question du genre, tout en maintenant un agenda critique et/ou féministe. Ces recherches vont toucher presque tous les domaines des sciences du langage, en se déployant aussi bien autour 
des questions de performance langagière et d'interaction qu'autour d'une lecture critique des grammaires, en passant par la sociophonétique, la rhétorique, l'analyse de discours, la syntaxe, la lexicographie, la sémantique, la sociolinguistique, la linguistique de corpus, ou encore la didactique.

11 Des ouvrages collectifs voient alors le jour ; trois d'entre eux, en particulier, marquent un tournant dans le domaine en lui donnant une visibilité : Langage, genre et sexualité (Duchêne \& Moïse 2011) en sociolinguistique, Intersexions, langues romanes, langue et genre (Baider \& Elmiger 2012) en linguistique historique et synchronique, et La face cachée du genre. Langage et pouvoir des normes (Chetcuti \& Greco 2012) principalement en analyse de discours et des interactions. Ces ouvrages rassemblent des travaux fondateurs, parfois méconnus, de chercheur.es reconnu.es comme de jeunes chercheur.es. Ils vont permettre de tracer une généalogie des recherches sur le genre, les sexualités et le langage. Suivront des numéros spéciaux de revue, principalement en sociolinguistique et en analyse du discours, comme Langage et société (Greco éd. 2014 : 148 et 2015 : 152), Les Cahiers de Linguistique (Tomc, Totozani, Ranchon éds. $2014: 40$ (1)), Synergies Italie (Nugara éd. $2014: 10$ ), puis Itinéraires (Paveau et Pahud éds. 2017), Mots. Les langages du politique (Chanay, Chevalier \& Gardelle éds. 2017), Semen (Abbou, Arnold et Marignier éds. 2017). En 2013, l'association Genres, Sexualités, Langage est créée à partir du réseau Genre et Langage né en 2009, animant une liste mail, un carnet de recherches, et organisant des événements en lien avec ces questions. Last but not least, on recense également plus d'une quinzaine de thèses en cours dans différentes universités dont l'articulation des questions de genre et du langage est le sujet principal : autant de travaux qui révèlent la vitalité de ce champ et son potentiel pour le futur paysage de la recherche.

Le constat est un peu différent en ce qui concerne les rapports entre littérature, genre et sexualités. Issues des mouvements féministes français des années 1960-1970, trois directions principales de recherches ont émergé : les réécritures de l'histoire littéraire depuis le point de vue des femmes, les travaux portant sur la différence sexuelle et enfin l'interrogation du genre par l'écriture littéraire.

13 La publicisation de voix féminines et féministes a en effet provoqué un mouvement de recherche et de redécouverte d'autres voix féminines dans l'histoire et dans la littérature (Duby \& Perrot 1991-1992, Leduc 2004), qui ont donné lieu à des anthologies (Moulin 1975, Mistacco 2005), à des projets éditoriaux (les éditions Des Femmes, créées en 1974 par A. Fouque ; collection Des Deux sexes et autres, PSE), à des études sur la figure de la femme écrivain (Planté 2015) et sur les écrivaines, et même à un Dictionnaire universel des créatrices (Calle-Gruber, Fouque \& Didier 2013). Cette démarche présente l'avantage de repenser le "patrimoine " littéraire à l'aune du féminisme, mais elle constitue davantage un travail historique qu'une réflexion sur la langue littéraire.

Par ailleurs, avec le groupe Psych \& Po (Psychanalyse et Politique), rattaché au MLF, une volonté de lier féminisme et littérature par le biais de la psychanalyse a émergé : c'est l'approche adoptée par des penseuses comme Julia Kristeva, Marcelle Marini, Hélène Cixous (qui forgea le concept d'« écriture féminine ", Cixous 2010) ou encore Luce Irigaray. Si elle lie écriture, féminité et féminisme (Revue des sciences humaines 1977), cette approche se préoccupe davantage du «féminin » que du "genre » et reste fondamentalement différentialiste. Genre(s) et différence sexuelle sont néanmoins pensés ensemble dans des travaux postérieurs (Negrón 1994, Calle-Gruber 2006, CalleGruber \& Germain 2006) qui privilégient la langue et s'éloignent du paradigme 
psychanalytique. Travaillant les genres (littéraire, «sexuel», grammatical) et non le genre (Frantz 2008; Frantz, Crevier-Goulet \& Calle-Gruber 2013), ces penseur.es s'inscrivent dans une démarche de déconstruction de la littérature, dont la voie a été ouverte par Jacques Derrida.

Enfin, le genre est au centre de deux approches principales : l'une, inspirée des travaux de Butler, aborde le genre comme un thème littéraire (voir par exemple Vannouvong 2010); l'autre s'inspire des travaux de Monique Wittig (2010) et travaille le genre comme concept en littérature. L'immense intérêt des travaux de Wittig réside dans l'ouverture de sa perspective, philosophique et littéraire, militante et théorique. Sans jamais céder au thématisme, puisqu'elle conçoit la langue comme un matériau, sans non plus perdre de vue la dimension politique et militante de la littérature, elle lie ainsi genre, sexualités et langue dans ses écrits théoriques comme dans ses récits.

On le voit, la question du genre a souvent été éclipsée dans le champ littéraire par la question des femmes et, en particulier, de l'écriture féminine. Pourtant, les pistes ouvertes par des auteures comme Wittig pour penser le genre en littérature trouvent aujourd'hui des continuateurs et continuatrices dans l'espace francophone (voir notamment Auclerc \& Chevalier 2012, Éribon 2015) qui, bien que marginaux.les, maintiennent un agenda scientifique vivace. Là encore, un lieu de visibilité semble nécessaire afin de fédérer ces travaux.

Dans le champ de la philosophie, les recherches féministes, ainsi que les travaux sur le genre et les sexualités ont été et restent souvent délégitimés et marginalisés, pour des raisons politiques et institutionnelles similaires aux autres disciplines, avec, peut-être, une résistance accrue de nombreux universitaires à interroger le rapport entre leurs productions philosophiques et leurs positionnements sociaux et politiques (c'est ce que mettent en évidence aussi bien Pierre Bourdieu lorsqu'il définit le champ scolastique dans les Méditations pascaliennes que les philosophes féministes qui ont travaillé sur le sexisme de la tradition philosophique (Le Dœuff 1989, Collin, Pisier \& Varikas 2000) ou les épistémologies féministes du point de vue (Harding 2003)).

Ces thématiques ont pourtant dessiné un champ complexe de recherches très différentes selon le prisme politique et féministe adopté. Inaugurée par Simone de Beauvoir (1949), qui dialogue avec la phénoménologie et l'existentialisme, une première approche de ces questions est à la fois anti-essentialiste et matérialiste. Développée par des auteures de différentes disciplines, comme Christine Delphy (1998, 2001), Colette Guillaumin (1992), Danièle Kergoat (2012), Nicole-Claude Mathieu (1991) ou Paola Tabet $(1998,2004)$, cette approche pose, dans la lignée du marxisme, la question des rapports de détermination entre l'infrastructure et la superstructure, donc entre la base matérielle (production, économie, etc.) et la culture, le savoir et, bien entendu, le langage. Parmi ces auteures, Monique Wittig a apporté une réponse originale à la question du langage comme reflet des rapports matériels ou comme étant lui-même matériel, en faisant du langage un espace d'action et de lutte et en affirmant, en même temps, la matérialité du langage et son rôle dans la constitution des rapports de genre et de l'hétéronormativité (Wittig 2001). Ces féministes matérialistes, de même que d'autres philosophes comme Françoise Collin, inscrivent leur recherche dans le militantisme féministe (comme le montrent les revues qu'elles éditent: Les cahiers $d u$ GRIF; Questions féministes, rééditée depuis 2001 sous le titre Nouvelles questions féministes). Dialoguant avec d'autres disciplines, comme la sociologie ou l'anthropologie, certaines recherches philosophiques contemporaines renouvellent 
aujourd'hui ce féminisme matérialiste depuis les études de genre et les études queer (voir par ex Cornelia Möser 2013, ainsi que le numéro de la revue Comment s'en sortir? consacré aux matérialismes féministes et dirigé par Maxime Cervulle et Isabelle Clair, à paraitre en décembre 2016) ou depuis les cultural studies et le renouvellement qu'elles apportent à la pensée matérialiste de la culture et du langage (voir par ex Cervulle, Quemener \& Vörös 2016).

Déjà évoquée plus haut dans le cadre de la littérature, une deuxième ligne d'analyse philosophique, différentialiste, est ouverte par les travaux de Julia Kristeva, Hélène Cixous ou Luce Irigaray. C'est avec ces auteures que débattent des philosophes étatsunien.nes comme Judith Butler, à l'origine d'une conceptualisation radicalement antiessentialiste du genre et des sexualités. Le développement d'une théorie critique du genre s'appuie ainsi sur ce qu'on a appelé la French Theory, donc, pour ce qui concerne la philosophie, sur les auteures citées précédemment, mais aussi sur la relecture du marxisme proposée notamment par Louis Althusser (sa conception de l'idéologie et sa notion d'interpellation (1982)) ; sur les travaux de Michel Foucault (son histoire de la sexualité ainsi que son travail sur la discursivité et sur la production des subjectivités dans et par les rapports de pouvoir $(1971,1976))$; sur le postmodernisme inspiré de Jacques Derrida, Jean-Luc Nancy ou Jean-François Lyotard (et notamment, concernant le langage, sur la remise en cause du structuralisme opérée par Derrida et la déconstruction des dualismes hiérarchisés qu'il véhicule (Derrida 1967)); sur la remise en question radicale du sujet, de l'individu et de l'identité opérée par Gilles Deleuze et Félix Guattari $(1972,1980)$. Elle s'appuie également sur la philosophie analytique et la philosophie du langage ordinaire inspirée de Ludwig Wittgenstein et John L. Austin (voir par exemple Ambroise \& Laugier 2009, 2011). En effet, si la philosophie analytique inspirée de Bertrand Russell et de Gottlob Frege cherche à clarifier le langage par la logique, la philosophie du langage ordinaire (le second Wittgenstein, Austin, Peter Frederick Strawson, Gilbert Ryle, etc.) recentre l'analyse sur les usages ordinaires du langage et sur les rapports entre le langage et les formes de vie (voir notamment Cavell 1979). C'est la théorisation par Austin de la performativité du langage, qui critique une conception purement référentialiste du langage et l'idée que la vérité est sa seule dimension d'analyse pour penser la parole comme acte (1975), qui a été la plus utilisée dans une perspective féministe et d'études de genre : pour penser le genre comme le produit d'une efficacité performative (Butler 1990) ou pour interroger la vulnérabilité des sujets au langage, le pouvoir blessant des insultes, la possibilité de la subversion ou la réduction au silence (comme le montrent les débats sur le 1er amendement de la Constitution des États-Unis, à propos de l'efficacité performative des discours de haine et des discours pornographiques: Judith Butler, Gayle Rubin, Andrea Dworkin, Rae Langton, Catharine MacKinnon, Jennifer Hornsby, Mary Kathryn McGowan. Voir notamment: MacKinnon 1993, Butler 1997). L'analyse butlerienne du genre comme performatif et la théorisation du queer par Teresa de Lauretis (2007) nourrissent à leur tour des recherches philosophiques anti-essentialistes et queer dans l'espace francophone (voir par exemple les travaux de Sam Bourcier (2001) et de Paul B. Preciado (2008), mais aussi plusieurs thèses en cours, la publication de la revue Comment s'en sortir? depuis 2015, etc.). Ces travaux partagent la remise en question postmoderne du sujet ainsi qu'une conception du langage comme performatif et producteur de la réalité sociale, notamment des rapports de genre et de sexualités. D'autres recherches philosophiques contemporaines vont partir de l'éthique du care pour analyser le silence, la vulnérabilité et leurs implications morales et politiques (par 
ex. Laugier \& Paperman 2005). Certains travaux, s'inscrivant dans une perspective de décolonisation de la pensée, posent la nécessité de faire une analyse intersectionnelle du langage et des rapports sociaux, qui peut s'appuyer à la fois sur les textes fondamentaux du Black Feminism (on peut penser par exemple aux textes de bell hooks sur le langage comme lieu de lutte (1990), ou à ceux d'Audre Lorde sur la poésie et la nécessité de transformer le silence en paroles et en actes (1984). Voir aussi l'anthologie établie par Dorlin en 2008) ainsi que sur des auteurs décoloniaux comme Frantz Fanon, Edward Saïd ou Gayatri Chakravorty Spivak (voir notamment Spivak 1988). Ces recherches héritent généralement des épistémologies féministes du point de vue et des savoirs situés (Sandra Harding, Donna Haraway, Patricia Hill Collins, etc. Voir Harding 2003), cherchent à rendre compte des rapports entre position sociale, production des subjectivités et discours théorique et, dans la lignée de Foucault, prennent acte de la nécessité de décloisonner les savoirs. La revue se propose donc comme un espace de diffusion de travaux féministes et critiques sur le genre et les sexualités trop souvent marginalisés, comme un outil de dialogue avec d'autres disciplines, et comme un lieu de visibilisation, d'invention et de rencontre pour les philosophies féministes contemporaines.

Tout comme la linguistique anglophone, l'anthropologie anglophone a su elle aussi explorer la question du genre dans ses dimensions langagières, que ce soit depuis l'anthropologie linguistique (par exemple les travaux de Nelson sur le traitement du genre dans les dynamiques de revitalisation du Lakota (2016) ou ceux d'Hadzantonis sur les discours LGBT en Asie orientale (2017)) ou depuis les cultural studies, qui font la part belle à la construction des récits dans les études de genre (comme le montre l'ouvrage de Clare Hemmings, Why Stories Matter : The Political Grammar of Feminist Theory (2011)) et portent une attention soutenue aux interactions de terrain au prisme de la sexualité (Giametta 2017, Raboin 2017). En France, bien que moins répandues, les recherches qui tentent de saisir le genre dans ses dimensions langagières existent également, enracinées dans l'anthropologie structuraliste (Godelier 1978) et l'anthropologie sociale. À la frontière entre linguistique et anthropologie, des travaux interrogent par exemple en quels mots des acteurs sociaux disent le genre, la sexualité ou encore l'amour (Miller \& Cheikh 2011, Pereira 2010). Ces recherches ont le mérite d'inscrire la dimension langagière dans ce qui fait culture et ce qui fait société.

21 Par ailleurs, la question du langage en anthropologie s'est traditionnellement posée dans les rencontres de la discipline avec la littérature (Leiris 1988, Geertz 1996, Geertz 1998, Bourdieu 2001, Boyer 2001, Yacine-Titouh 2010, Éribon 2009). C'est donc également par ce biais-là qu'aujourd'hui des recherches interrogent le genre et les sexualités dans un prisme anthropo-littéraire (Gouyon 2013a).

Au-delà de ces recherches sur les discours des acteurs, l'anthropologie, en raison de sa tradition réflexive, est aussi un lieu d'interrogation du discours et, plus particulièrement, du récit scientifique et de son rapport au réel. Les travaux qui s'inscrivent dans la lignée des études postcoloniales et décoloniales ne manquent pas de développer une approche critique de la fabrique du discours anthropologique et plus largement des sciences humaines et sociales, comme en témoigne "Décoloniser les savoirs » (Boulbina, Cohen, Zouggari \& Simon éds. 2012). Et cette interrogation touche bien sûr aux récits du genre et récits sur le genre en circulation dans le champ anthropologique (Éribon 2003, Moujoud \& Pourette 2005, Gouyon 2013b). Tout un champ de recherche anthropologique reste ainsi ouvert pour explorer et bâtir des liens 
entre discours et genre, en faisant dialoguer les différentes compréhensions du discours, de la langue et du genre en circulation dans différentes disciplines. La revue se propose d'être un espace pour cette rencontre à venir.

Pour finir ce tour d'horizon non-exhaustif des rencontres entre langage et genre, il faut bien sûr également mentionner les Sciences de l'Information et de la Communication, qui donnent depuis quelques années une place de choix à la question du genre dans les médias, mais également dans les mouvements politiques, les institutions politiques ou encore l'espace numérique. Des chercheursses telles que Kunert, Coulomb-Gully, Montanola, Quemener, Cervulle, pour ne mentionner qu'eulles, développent, à la frontière des cultural studies, des media studies, des digital studies, et de l'analyse de discours, des travaux qui interrogent directement la matérialité discursive du genre et des sexualités dans des corpus émergents. Les configurations qu'ils et elles analysent révèlent la diffusion mais aussi la construction et déconstruction permanente des catégories d'hommes et de femmes à travers les discours.

Enfin, le rapport entre langage et sexualités mériterait lui aussi un développement à part entière, qui sera l'objet d'un futur numéro de la revue.

Au-delà d'un objet de recherche commun et de l'accroissement du nombre de travaux, il apparaît donc que cette intersection entre genre, sexualités et langage a dessiné un espace de recherche protéiforme mais spécifique, dont les lignes transversales sont une compréhension critique de la langue et du discours comme objets polysémiques et non transparents, une prise en compte des rapports de pouvoir dans l'analyse linguistique, une prise en compte de la dimension agissante du langage sur les catégories de genre et - conséquemment - une lecture du genre non-essentialiste. Cette lecture commune du genre, des sexualités et du langage ainsi que de leurs intersections est en train de paver la route pour la constitution d'un domaine de recherches francophones.

On le voit, en fait de rendez-vous manqué, il s'agit davantage d'un manque de visibilité et d'un manque d'espace commun pour ces recherches. Il semble donc plus que jamais nécessaire de créer des dialogues et des liens par-delà le découpage disciplinaire et de renforcer ces dynamiques par la création d'un espace pérenne de discussion comme celui que peut offrir une revue. Car, malgré une activité foisonnante, il n'existait jusqu'ici aucun espace éditorial francophone au long cours dédié aux recherches portant sur le genre et les sexualités dans leurs dimensions langagières.

C'est donc pour répondre à cet éparpillement, à l'isolement des recherches et surtout à la visibilité lacunaire du domaine que GLAD! a été créée. La ligne scientifique de la revue est façonnée par ces modalités d'émergence du champ. Bien que le projet ait été initié par des linguistes, ce que montre l'état des lieux par discipline, c'est que les recherches critiques sur le genre, le langage et les sexualités sont déjà en dialogue par-delà les ancrages disciplinaires. C'est pourquoi la revue ne peut ni ne veut se limiter à la seule lecture disciplinaire : c'est en entrecroisant nos regards et nos savoirs de linguistes, d'anthropologues, de philosophes, d'analystes du discours et de littéraires - entre autres! - que nous saisirons et interrogerons les rapports entre langage, genre et sexualités. 


\section{Tenter une ligne éditoriale queer. Interdisciplinarité, francophonie et savoirs minoritaires}

Le projet de la revue est donc de créer un espace de réflexion pluridisciplinaire, ou plutôt « indisciplinée », permettant de prendre en compte les rapports de pouvoir dans l'analyse linguistique, la dimension agissante du langage sur les catégories de genre, et de développer une lecture du genre non essentialiste et intersectionnelle. Il s'agit d'explorer la manière dont le langage construit, représente, performe, déstabilise, subvertit, rend possibles ou impossibles les rapports de genre et de sexualité, tout en prenant la mesure de ce que ces analyses mettent au jour quant aux langues et aux discours.

Prendre au sérieux un tel projet demande alors une attention permanente à nos propres modalités de création et diffusion des discours. C'est en ce sens que les six principes de la ligne éditoriale ont été dessinés. Il s'agit de la valorisation des recherches minoritaires et/ou menées depuis les marges (y compris la jeune recherche), de la transdisciplinarité, d'une ouverture à toutes les francophonies, d'une réflexion sur les modalités de production et de diffusion des savoirs contemporains sur le genre, d'une attention portée à la multiplicité des possibilités d'écriture du genre et enfin du choix de la diffusion en libre accès. Allier ces différentes dimensions nous semble permettre d'ébaucher une ligne éditoriale féministe et queer, en ce qu'elle travaille les zones hybrides de la production du savoir, et cherche à décatégoriser les types de discours pour ouvrir de nouvelles formes, et donc de nouvelles façons de penser le genre et le langage.

\section{Visibiliser les recherches minoritaires}

GLAD! s'ouvre volontiers aux recherches minoritaires, et se propose d'être une tribune pour de tels travaux. Par recherches minoritaires, nous entendons des recherches portant sur des sujets "illégitimes", des recherches produites depuis des espaces marginaux, des recherches critiques sur ce qui est minorisé et sur les phénomènes de minorisation, de mise à la marge, mais aussi des recherches menées dans des formats exploratoires (audio, vidéo, travail collectif, etc.) qui questionnent les façons traditionnelles d'énoncer la pensée. Cela dessine une sorte de recherche mineure qui puisse servir de contrepoint aux discours les plus diffusés.

31 La revue souhaite aussi encourager la publication des travaux issus de la jeune recherche - particulièrement dynamique, avec de plus en plus de thèses consacrées aux questions de genre, de sexualités et de langage. La revue compte ainsi parmi ses objectifs de diffuser les travaux les plus récents ainsi que les approches innovantes, proposant de nouveaux cadres, de nouveaux terrains ou de nouvelles intersections théoriques.

\section{Indiscipline et exploration}

GLAD! veut promouvoir une véritable pluridisciplinarité et encourager l'intégration d'analyses langagières dans d'autres disciplines que la linguistique ainsi que le développement d'un prisme critique sur le genre et les sexualités dans toutes les disciplines. Cette question se pose peut-être de manière accrue pour la linguistique, du 
fait d'une tradition sociologique et philosophique majoritaire en études de genre. La revue propose de développer une double expertise sur ces questions, et non simplement d'intégrer le genre comme une variable dans le langage, ni le langage comme une simple dimension du genre. On pourra comprendre le langage dans ses dimensions discursives, textuelles, morphosyntaxiques, phonétiques, littéraires, pragmatiques, sémiotiques, etc., et le genre et les sexualités dans leurs dimensions relationnelles, identitaires, structurelles, idéologiques, matérielles, etc.

A la fois outils d'analyse, angles d'attaque et objets de recherche, le genre, les sexualités et le langage sont par nature interdisciplinaires. Les associer peut donc permettre d'éviter l'enfermement disciplinaire. Plus encore, au-delà de la représentation pluridisciplinaire, la revue encourage l'exploration d'une transdisciplinarité, qui fasse dialoguer au cœur des articles différents champs et points de vue. Pour ce faire, les articles transdisciplinaires bénéficient d'un format plus long afin de pouvoir y déployer et $\mathrm{y}$ articuler autant d'outils théoriques et méthodologiques que nécessaires au développement d'une pensée transversale.

La revue encourage par ailleurs une écriture qui questionne le cloisonnement disciplinaire pour favoriser la circulation des savoirs et créer un espace ouvert au dialogue. Elle est ouverte à différentes approches théoriques et méthodologiques, et ne représente pas une école de pensée. Elle cherche au contraire à valoriser la multiplicité des points de vue, et à fournir un panorama élargi des recherches existantes, à la condition d'une perspective émancipatrice, impliquant une réelle prise en compte des savoirs situés.

La revue se caractérise également par sa volonté d'articuler des recherches académiques à des travaux artistiques et politiques abordant eux aussi la question du langage, du genre et/ou des sexualités. Cette rencontre peut permettre non seulement de pluraliser les approches, les supports et les modalités d'analyse des thématiques de la revue, mais aussi de rendre compte d'un dialogue déjà existant entre les sphères académiques, militantes et artistiques, voire de renforcer ce dialogue et d'en réaffirmer la nécessité.

\section{Francophonies}

La question de la langue de publication nous importe bien sûr particulièrement. La revue s'adresse à l'ensemble de la communauté francophone, de tous les continents, dans toute sa pluralité. Ainsi, des travaux francophones portant sur d'autres espaces que la France et prenant comme objet différentes langues sont les bienvenus. Par ailleurs, la revue n'attend pas nécessairement un français "natif ", dont on ne saurait de quel espace il serait natif. Le critère principal sera celui de la pertinence scientifique.

\section{Poser des bases féministes au travail éditorial}

37 La revue souhaite offrir aux contributeurices un espace bienveillant et accompagnant. Elle s'est notamment dotée d'une charte éthique à laquelle souscrivent les évaluateurices et les membres du comité de rédaction: prenant acte du caractère excluant de certains codes et hiérarchies académiques, du sentiment d'illégitimité qu'ils peuvent construire et de la façon dont ils peuvent délégitimer certains savoirs, 
cette charte a été pensée pour fragiliser, dans la mesure du possible, de tels effets indissociablement sociaux et académiques.

\section{Multiplier nos façons d'écrire le genre} que d'une volonté d'accueillir au sein de la revue différentes perspectives contemporaines travaillant genre, langage et sexualités. Ce premier numéro varia se veut donc ouvert, pluriel, tout en affirmant une conception commune du genre comme construit par et à travers les discours, et ce dans les trois rubriques qui organisent la revue, « Recherches », « Explorations » et « Créations ». Des lignes de force se dessinent ainsi au-delà des différences d'approche entre les articles de ce numéro.

D'abord, c'est une idéologie humaniste qui est mise en défaut, idéologie héritée des Lumières et du xvIII ${ }^{e}$ siècle français, et qui masque les rapports sociaux de sexe sous une conception universaliste de l'humain ; Nadia Ouabdelmoumen («Genre, migrations : l'autonomie à l'épreuve du volet linguistique du contrat d'accueil et d'intégration ») critique la notion d'autonomie dans cette perspective, et montre comment la langue et son acquisition, présentées comme des moteurs d'émancipation pour les migrant.es, jouent, au sein du dispositif lié au "contrat d'accueil et d'intervention », un rôle de perpétuation des rapports sociaux et de genre, et conséquemment du racisme et du sexisme. Aron Arnold («Idéologies de genre et construction des savoirs en sciences phonétiques») met lui aussi au jour les impensés de «l'idéologie de genre » binaire 
associant le masculin à l'universel, lorsqu'il déconstruit ses effets au sein des savoirs produits par les sciences phonétiques. Il démontre comment cette universalisation du masculin pathologise le féminin et cadastre les corps pour construire un système différencié binaire, lieu même de la construction du genre sous l'autorité du discours scientifique et de son objectivité prétendue. Dans la même perspective de critique des savoirs dominants sur les corps, Hélène Mourrier («TRANS-formation : FT* \& MT*») revient sur un travail réalisé avec l'association OUTrans autour de livrets d'information sur les modifications corporelles qui peuvent être réalisées sur les personnes trans*, dont nous publions quatre planches. Elle explicite les choix qu'elle a mis en œuvre dans son travail graphique afin de produire des documents pédagogiques qui ne reprennent pas l'iconographie médicale. Elle explore par là les enjeux de la représentation sémiotique des corps trans* pour la construction des savoirs et des réappropriations corporelles. Enfin, dans la lignée de ces travaux critiques, Anne-Laure Garcia ( $\mathrm{La}$ Protection Maternelle. Catégorisations et hiérarchisations du féminin dans les discours juridiques d'Après-guerre ») interroge ce qui se cache sous la notion juridique de "protection maternelle ", en mettant en œuvre une démarche distanciatrice qui porte sur les dimensions à la fois historique et discursive du droit. La perspective comparative qu'elle adopte lui permet ainsi de montrer que dans les trois espaces observés que sont la RDA, la RFA et la France de l'après-guerre, la protection maternelle peut rapidement dériver vers une protection contre la mère, qui devient alors une figure ambiguë et peut menacer tout autant que protéger.

Ces travaux ont donc en partage d'insister sur la co-construction permanente du genre et des discours. Le roman Pénis d'orteil de Matsuura Rieko offre l'occasion à Alexandre Taalba, dans un compte-rendu critique, de réfléchir à ces co-constructions, particulièrement saillantes dans un ouvrage mettant en scène une jeune femme se trouvant un matin affublée d'un pénis à la place du gros orteil. Comment dire le sexe, la sexualité, le rapport sexuel lorsqu'une telle mutation a eu lieu? Cette transformation imaginaire, dans le bouleversement qu'elle fait subir à nos catégories de genre, de sexe, de sexualités, informe le rapport entre genre et langage.

Pour finir, trois travaux s'intéressent à des modes de réinvention de soi à l'écart ou à l'encontre de l'«idéologie de genre» dominante. Catherine Gonnard et Elisabeth Lebovici ( Inventer son genre dans le langage de la télévision ») montrent comment une culture lesbienne dissidente, minoritaire, s'est élaborée et rendue paradoxalement visible dans la France de l'avant "libération sexuelle", dans des émissions de divertissement télévisuelles, en particulier Cabaret du soir. Autre contre-culture, le voguing fait l'objet d'une série de photographies de Lila Neutre, ainsi que d'un texte ("VOGUE-rs ») : danse et pratique apparue dans le milieu gay Noir new-yorkais des années 1980, et rendue célèbre par le film controversé de Jennie Livingston Paris is Burning, le voguing suscite aujourd'hui un regain d'intérêt au sein de la culture mainstream; les photographies de Lila Neutre semblent néanmoins suggérer que la multiplication des catégories qu'il encourage, le jeu qui en fait toute la saveur, la transformation permanente de soi qu'implique le voguing ne se laissent pas aisément approprier par une culture dominante, hétérosexuelle, cis et blanche. Aux côtés de ces photos, la rubrique "Créations » accueille également Eau'Rageuses, groupe de musique féministe. Le poème Corpo, écrit par Catarina Fernandes et mis en musique par les Eau'Rageuses, est en écoute, accompagné de son texte bilingue en français et portugais. Ce travail des langues autour du corps - et sa mise en musique - soulève des questions 
sur les possibilités de dire le corps qui tente de s'échapper à ce qu'on lui impose d'être, en se glissant dans l'interstice de l'interlangue.

\section{BIBLIOGRAPHIE}

ABBOU, Julie. 2011. L'antisexisme linguistique dans les brochures libertaires : pratiques d'écriture et métadiscours. Thèse de doctorat en sciences du langage. Marseille : Université d'Aix-Marseille. ABBOU, Julie, ARNOLD, Aron \& MARIGNIER, Noémie (éds.). 2017. Semen. Numéro spécial Le genre, lieu de l'hétérogène.

ALTHUSSER, Louis. 1982. Positions. Paris : Éditions sociales.

AMBROISE, Bruno \& LAUGIER, Sandra. 2009. Philosophie du langage. Tome 1, «Signification, vérité, réalitê ». Paris : Vrin.

AMBROISE, Bruno \& LAUGIER, Sandra. 2011. Philosophie du langage. Tome 2, « Sens, usage et contexte ». Paris : Vrin.

ARMSTRONG, Nigel, BEAUVOIS, Cécile \& BEECHING, Kate. 2001. La langue française au féminin. Le sexe et le genre affectent-ils la variation linguistique? Paris : L'Harmattan.

ARNOLD, Aron. 2008. « Genre et langage », Forum für Politik, Gesellschaft und Kultur, 277 : 33-35.

ARNOLD, Aron. 2015. La voix genrée, entre idéologies et pratiques. Une étude sociophonétique. Thèse de doctorat en phonétique. Paris : Université Sorbonne Nouvelle-Paris 3.

AUCLERC, Benoît \& CHEVALIER, Yannick (éds.). 2012. Lire Monique Wittig aujourd'hui. Lyon :

Presses universitaires de Lyon.

AUSTIN, John Langshaw. 1975. How to do things with words : the William James lectures delivered at Harvard University in 1955. Cambridge : Harvard University Press.

BAIDER, Fabienne. 2004. Hommes Galants, Femmes Faciles - Études Socio-Sémantique et diachronique. Paris : L'Harmattan.

BAIDER, Fabienne \& ELMIGER, Daniel. 2012. Intersexion. Munich : Lincom.

BAILLY, Sophie. 2008. Les hommes, les femmes et la communication : Mais que vient faire le sexe dans la langue. Paris : L'Harmattan.

BAKER, Paul. 2008. Sexed Texts : Language, Gender and Sexuality. London : Equinox.

BEAUVOIR, Simone (de). 1949. Le deuxième sexe. Paris : Gallimard.

BOULBINA, Seloua Luste, COHEN, Jim, ZOUGGARI, Najate \& SIMON, Patrick (éds.). 2012.

Mouvements. Numéro spécial Décoloniser les savoirs. Internationalisation des débats et des luttes.

BOURCIER, Sam. 2001. Queer zones. Tome 1, « Politiques des identités sexuelles, des représentations et des savoirs ». Paris : Balland.

BOURDIEU, Pierre. 1997. Méditations pascaliennes. Paris : Seuil.

BOURDIEU, Pierre. 2001. Langage et pouvoir symbolique. Paris : Seuil. 
BOYER, Pascal. 2001. Et l'homme créa les dieux : Comment expliquer la religion (trad. Claude-Christine Farny). Paris : Robert Laffont.

BREYSSE, Chrystel. 2002. Du général aux particulières : politiques linguistiques de féminisation de la langue en France, Belgique, Suisse et Québec. Thèse de doctorat en sciences du langage. Aix-enProvence : Université de Provence.

BURTON, Deirdre. 1982. «Through glass darkly : through dark glasses », in Literature and Language : an Introduction Reader in Stylistics, Carter, Ronald (ed.). London : George Allen and Unwin.

BUTLER, Judith. 1990. Gender Trouble. New York : Routledge.

BUTLER, Judith. 1997. Excitable speech : a politics of the performative. New York : Routledge.

CALLE-GRUBER, Mireille (éd.). 2006. Littérature, 146. La différence sexuelle en tous genres. Paris : Larousse.

CALLE-GRUBER, Mireille \& GERMAIN, Marie-Odile (éds.). 2006. Genèses, généalogies, genres : autour de l'œuvre d'Hélène Cixous. Actes du colloque tenu à la Bibliothèque nationale de France du 22 au 24 mai 2003. Paris : Galilée.

CAMERON, Deborah. 1990. The Feminist critique of language : a reader. London ; New York: Routledge.

CAMERON, Deborah. 1992. Feminism and Linguistic Theory. London : Macmillan.

CAMERON, Deborah \& KULICK, Don. 2006. The Language and Sexuality Reader. London ; New York : Routledge.

CAVELL, Stanley. 1979. The claim of reason: Wittgenstein, skepticism, morality, and tragedy. Oxford : Clarendon Press.

CERVULLE, Maxime \& CLAIR, Isabelle (éds.). 2016. Comment s'en sortir ? Numéro spécial Matérialismes féministes.

CERVULLE, Maxime, QUEMENER, Nelly \& VÖRÖS, Florian. 2016. Matérialismes, culture \& communication. Tome 2, «Cultural Studies, théories féministes et décoloniales ». Paris : Presses des Mines.

CHANAY, Hugues (de), CHEVALIER, Yannick \& GARDELLE, Laure (éds.). 2017. Mots. Les langages du politique. Numéro spécial Écrire le genre.

CHETCUTI, Nadia \& GRECO, Luca (éds.). 2012. La Face cachée du genre. Langage et pouvoir des normes. Paris : Presses Sorbonne Nouvelle.

CIXOUS, Hélène. 2010. Le Rire de la Méduse et autres ironies. Paris : Galilée.

COATES, Jennifer \& PICHLER, Pia. 2011 [1998]. Language and gender : a reader. Malden : WileyBlackwell.

COLLIN, Francoise, PISIER, Evelyne \& VARIKAS, Eleni. 2000. Les femmes, de Platon à Derrida : anthologie critique. Paris : Plon.

DE LAURETIS, Teresa. 2007. Théorie queer et cultures populaires : de Foucault à Cronenberg (trad. Sam Bourcier). Paris : La Dispute.

DELEUZE, Gilles \& GUATTARI, Félix. 1972. Capitalisme et schizophrénie. L'anti-Edipe. Paris : Éditions de minuit.

DELEUZE, Gilles \& GUATTARI, Félix. 1980. Mille plateaux. Paris : Éditions de minuit. 
DELPHY, Christine. 1998. L'ennemi principal. Tome 1, « Economie politique du patriarcat ». Paris : Syllepse.

DELPHY, Christine. 2001. L'ennemi principal. Tome 2, « Penser le genre ». Paris : Syllepse.

DELPHY, Christine. 2008. Classer, dominer. Qui sont les autres. Paris : La Fabrique.

DERRIDA, Jacques. 1967. De la Grammatologie. Paris : Éditions de Minuit.

DIDIER, Béatrice, FOUQUE, Antoinette \& CALLE-GRUBER, Mireille. 2013. Le Dictionnaire universel des créatrices. Paris : Des Femmes.

DORLIN, Elsa. 2008. Black feminism : anthologie du féminisme africain-américain, 1975-2000. Paris :

L'Harmattan.

DUBY, Georges \& PERROT, Michelle. 1991-1992. Histoire des femmes en Occident. Paris : Plon.

DUCHÊNE, Alexandre \& MOÏSE, Claudine. 2011. Langage, genre et sexualité. Montréal : Nota Bene.

ECKERT, Penelope \& MCCONNELL-GINET, Sally. 2003. Language and Gender. Cambridge : Cambridge University Press.

ÉRIBON, Didier. 2003. Hérésies : essais sur la théorie de la sexualité. Paris : Fayard.

ÉRIBON, Didier. 2009. Retour à Reims. Paris : Fayard.

ÉRIBON, Didier. 2015. Théories de la littérature. Système du genre et verdicts sexuels. Paris : PUF.

FOUCAULT, Michel. 1971. L'ordre du discours : leçon inaugurale au Collège de France prononcée le 2 décembre 1970. Paris : Gallimard.

FOUCAULT, Michel. 1976. Histoire de la sexualité. Tome 1, « La volonté de savoir ». Paris : Gallimard.

FRANTZ, Anaïs. 2008. «Profession de foi d'un Centre de Recherches en Études Féminines et de Genres », Sens Public [En ligne], consulté le 11 novembre 2016.

FRANTZ, Anaïs, Crevier-Goulet Sarah-Anaïs \& Calle-Gruber, Mireille (éds.). 2013. Fictions des genres. Dijon : Éditions universitaires de Dijon.

Geertz, Clifford. 1996. Ici et là-bas : l'anthropologue comme auteur (trad. Daniel Lemoine). Paris : Métaillé.

GEERTZ, Clifford (et al.). 1998. La description. Paris : Éditions Parenthèses.

GIAMETTA, Calogero. 2017. The Sexual Politics of Asylum : Sexual Orientation and Gender Identity in the UK Asylum System. London; New-York : Routledge.

GODELIER, Maurice. 1978. «Les rapports hommes-femmes : le problème de la domination masculine ", in La Condition féminine, Centre d'études et de recherches marxistes (éd.). Paris : Éditions sociales, 23-44.

GOUYON, Marien. 2013a. « La ruse dans la transaction sexuelle : une liaison constitutive des rapports sociaux homosexuels de milieu populaire à Casablanca ", Pensée Plurielle, 33-34 : 217-226. GOUYON, Marien. 2013b. « Abdellah Taïa et "l'ethnologie de soi-même”. Du point de vue de l'objet à la construction de l'objet », Tumultes, 41 : 185-204.

GRECO, Luca. 2014. Langage et Société, 148. Numéro spécial Recherches linguistiques du le genre : bilan et perspectives.

GRECO, Luca. 2015. Langage et Société, 152. Numéro spécial Genre, langage et sexualité : données empiriques. 
GUILLAUMIN, Colette. 1992. Sexe, race et pratique du pouvoir : l'idée de nature. Paris : Côté-femmes. GUIRAUD, Pierre. 1978. Sémiologie de la sexualité. Paris : Payot.

HADZANTONIS, Michael D. 2017. The Linguistic Anthropologies of LGBT communities in East Asian regions : Developing and contextually Framing the Vergence Theory. New York : Taylor and Francis.

HARAWAY, Donna. 1988. «Situated Knowledge : The Science Question in Feminism and the Privilege of Partial Perspective », Feminist Studies, 14(3) : 575-599.

HARDING, Sandra G. (éd.). 2003. The feminist standpoint theory reader : intellectual and political controversies. New York : Routledge.

HEMMINGS, Clare. 2011. Why stories matter : the political grammar of feminist theory. Durham : Duke University Press.

HOLMES, Janet \& MEYERHOFF, Miriam. 2003. The Handbook of Language and Gender. Oxford: Blackwell.

hooks, bell. 1990. Yearning : race, gender, and cultural politics. Boston, MA : South End Press.

HOUDEBINE, Anne-Marie. 1989. «L'une n'est pas l'autre, ou Genre et sexe en français contemporain », in Linx [En ligne], 21(1), consulté le 11 novembre 2016. URL : www.persee.fr/doc/ linx_0246-8743_1989_num_21_1_1135.

HOUDEBINE, Anne-Marie. 2003. « Trente ans de recherche sur la différence sexuelle, ou Le langage des femmes et la sexuation dans la langue, le discours, les images ", Langage et Société, $106: 33-61$.

KERGOAT, Danièle. 2012. Se battre, disent-elles. Paris : La Dispute.

KHAZNADAR, Edwige. 2002. Le Féminin à la française : académisme et langue française. Paris :

L'Harmattan.

LAUGIER, Sandra \& PAPERMAN, Patricia. 2005. Le souci des autres : éthique et politique du care. Paris : Éditions de l'EHESS.

LE DEUUF, Michèle. 1989. L'Étude et le Rouet. Paris : Seuil.

LEDUC, Guyonne. 2004. Nouvelles sources et nouvelles méthodologies de recherche dans les études sur les femmes. Paris : L'Harmattan.

LEIRIS, Michel. 1988. L'Afrique fantôme. Paris : Gallimard.

LIVIA, Anna \& HALL, Kira. 1997. Queerly Phrased : Language, Gender, and Sexuality. Oxford : Oxford University Press.

LORDE, Audre. 1984. Sister outsider : essays and speeches. Trumansburg, NY : Crossing Press.

MACKINNON, Catharine A. 1993. Only Words. Cambridge : Harvard University Press.

MATHIEU, Marie-Jo. 2002. Extension du féminin : les incertitudes de la langue. Paris : Honoré Champion.

MATHIEU, Nicole-Claude. 1991. L'anatomie politique : catégorisations et idéologies du sexe. Paris : Côté - femmes.

MICHARD, Claire. 2002. Le Sexe en linguistique. Sémantique ou zoologie ? Paris : L'Harmattan.

MICHARD, Claire \& RIBERY, Claudine. 2008. Sexisme et sciences humaines, Pratique linguistique du rapport de sexage. Villeneuve d'Ascq : Presses Universitaires du Septentrion. 
MICHARD, Claire, \& VIOLLET, Catherine. 1991. « Sexe et genre en linguistique. Quinze ans de recherches féministes aux États-Unis et en R.F.A », Recherches Féministes, 4(2) : 97-128.

MILLER, Catherine \& CHEIKH, Myriam. 2011. « Les mots d'amour : Dire le sentiment et la sexualité au Maroc. De quelques matériaux », Estudios de Dialectologia Norteafricana y Andalusi, 13 : 173-199.

MILLS, Sara. 1995. Feminist Stylistics. London, New York : Routledge.

MILLS, Sara. 2008. Language and Sexism. Cambridge : Cambridge University Press.

MISTACCO, Vicky. 2005. Les femmes et la tradition littéraire : une anthologie du Moyen Âge à nos jours. New Haven : Yale University Press.

MÖSER, Cornelia. 2013. Féminismes en traductions : théories voyageuses et traductions culturelles. Paris : EAC.

MOTSCHENBACHER, Heiko. 2010. Language, Gender and Sexual Identity : Poststructuralist perspectives. Amsterdam ; Philadelphia : John Benjamins.

MOUJOUD, Nasima \& POURETTE, Dolorès. " “Traite” de femmes migrantes, domesticité et prostitution. À propos de migrations interne et externe », Cahiers d'études africaines [En ligne] 179-180, consulté le 10 novembre 2016. URL : www.cairn.info/revue-cahiers-d-etudesafricaines-2005-3-page-1093.htm.

MOULIN, Jeannine. 1975. Huit siècles de poésie féminine 1170-1975. Paris : Seghers.

NEGRÓN, Mara (dir.). 1994. Lectures de la différence sexuelle. Actes du colloque organisé par le Centre d'études féminines de l'Université de Paris-VIII et le Collège international de philosophie, 18-20 octobre 1990, Paris. Paris : Des femmes.

NELSON, Jessica Fae. 2016. « Lakota Men's and Women's speech. Gender, metapragmatic discourse, and language revitalization », Pragmatics and Beyond New Series, 264 : 227-254.

NUGARA, Silvia. 2014. Synergies Italie, 10. Numéro spécial Les discours institutionnels au prisme du "genre " : perspectives italo-françaises.

PAVEAU, Marie-Anne \& PAHUD, Sophie (éds.). 2017. Itinéraires. Numéro spécial Féminismes quatrième génération. Textes, corps, signes.

PEREIRA, Christophe. 2010. Le parler arabe de Tripoli, Lybie. Zaragoza : Instituto de Estudios Islámicos y del Oriente Próximo.

PERRY, Véronique (éd.). 2004. Actes du $3^{\circ}$ Colloque Internationale de Recherches Féministes Francophones : Désexisation et parité linguistique. Toulouse : ANEF

PERRY, Véronique. 2011. Aspects du genre dans la didactique de l'anglais. Thèse de doctorat en langues et littératures anglaises et anglo-saxonnes. Toulouse : Université Toulouse III-Paul Sabatier.

PLANTÉ, Christine. 2015 [1989]. La petite sœur de Balzac. Essai sur la femme auteur. Lyon : Presses universitaires de Lyon.

PRECIADO, Paul B. 2008. Testo junkie : sexe, drogue et biopolitique. Paris : Grasset.

Revue des Sciences Humaines. 1977. Écriture, féminité, féminisme, 168. Villeneuve d'Ascq : Presses universitaires du Septentrion.

RABOIN, Thibaut. 2017. Discourses on LGBT Asylum in the UK: Constructing a Queer Haven. Manchester : Manchester University Press. 
RIBERY, Claudine. 1981. «Rapport de sexage et repérages énonciatifs ou Femmes et hommes appartiennent-ils au genre humain ?», Pénélope, 4 : 68-70.

SCOTT, Joan W. 1986. « Gender : A Useful Category of Historical Analysis », The American Historical Review, 91(5) : 1053-1075.

SPIVAK, Gayatri Chakravorty. 1988. Can the subaltern speak? Basingstoke : Macmillan.

TABET, Paola. 1998. La construction sociale de l'inégalité des sexes : des outils et des corps. Paris :

L'Harmattan.

TABET, Paola. 2004. La grande arnaque : sexualité des femmes et échange économico-sexuel. Paris : L'Harmattan.

Talbot, Mary. 2010 [1996]. Language and Gender. Cambridge : Polity Press.

TOMC, Sandra, TOTOZANI, Marina \& RANCHON, Grâce. 2014. Cahier de linguistique. Numéro spécial Genres, Langues et Pouvoirs.

Vannouvoung, Agnès. 2010. Jean Genet : les revers du genre. Dijon : les Presses du réel.

VIENNOT, Éliane. 2014. Non, le masculin ne l'emporte pas sur le féminin! Paris : iXe.

VIOLI, Patrizia. 1987. « Les origines du genre grammatical », Langages [En ligne], 21(85), consulté le 11 novembre 2016. URL : www.persee.fr/doc/lgge_0458-726x_1987_num_21_85_1526.

WITTIG, Monique. 2001. La pensée straight. Paris : Baland.

WITTIG, Monique. 2010. Le chantier littéraire. Lyon : Presses universitaires de Lyon.

YACINE-TITOUH, Tassadit. 2010. Chacal ou la ruse des dominés. Paris : La Découverte.

YAGUELLO, Marina. 1989. Le sexe des mots. Paris : Belfond.

\section{AUTEURS}

\section{JULIE ABBOU}

Titulaire d'un doctorat de Sciences du Langage (Aix-Marseille Université) sur les modifications du genre linguistique pour des motifs politiques, Julie Abbou mène des recherches sur les apports théoriques réciproques des études de genre et des sciences du langage. Elle travaille également sur les dimensions sémiotiques du genre grammatical, ainsi qu'en rhétorique sur le traitement du genre dans différents types de discours. Récemment, elle a co-dirigé l'ouvrage Gender, Language and the Periphery. Grammatical and social gender from the margins (John Benjamins) et publié des articles dans des revues telles que Semen, Mots les langages du politique, Current issues in Language Planning, etc. Elle enseigne actuellement (2017) la linguistique à l'Université Paris 13.

\section{MARIA CANDEA}

Maria Candea, sociolinguiste et sociophonéticienne, s'intéresse principalement au français contemporain parlé en France. Maitresse de conférences à l'Université Paris 3 Sorbonne nouvelle

\section{MONA GÉRARDIN-LAVERGE}

Mona Gérardin-Laverge est doctorante en philosophie contemporaine à l'Université Paris 1 Panthéon Sorbonne. Sa thèse s'intitule « Language is also a place of struggle » (bell hooks), philosophie du langage ordinaire et féminisme. 


\section{STAVROULA KATSIKI}

Stavroula Katsiki est maîtresse de conférences en ethnolinguistique à l'université Paris 8 Vincennes Saint-Denis. Ses recherches, centrées sur la question de la diversité linguistique et culturelle, relèvent de la pragmatique contrastive, du plurilinguisme littéraire et de la traduction.

\section{NOÉMIE MARIGNIER}

Noémie Marignier est docteure en Sciences du Langage et Attachée Temporaire d'Enseignement et de Recherche à l'université Paris 3 Sorbonne Nouvelle. Ses travaux de recherche portent sur l'articulation entre corps/sexe/sexualité et discours. Elle a soutenu en 2016 une thèse en analyse $\mathrm{du}$ discours intitulée Les matérialités discursives du sexe. La construction et la déstabilisation des évidences du genre dans les discours sur les sexes atypiques. Elle est membre associée du laboratoire Clesthia (Université Paris 3 Sorbonne Nouvelle) ainsi que de Pléiade (Université Paris 13 Sorbonne Cité).

\section{LUCY MICHEL}

Lucy Michel est docteure en linguistique française et ATER à l'Université de Bourgogne. Ses travaux sont centrés sur la question du genre grammatical des dénominations de la personne en langue française, notamment d'un point de vue sémantique. Elle a soutenu une thèse sur la question en 2016.

\section{CHARLOTTE THEVENET}

Doctorante en littérature française à University College London (SELCS, French), Charlotte Thevenet prépare une thèse sur la rhétorique du commentaire chez Jacques Derrida. Mots-clefs : rhétorique, argumentation, commentaire. 